\title{
Linear independence of values of Tschakaloff series
}

\author{
K. Väänänen and V.V. Zudilin
}

In this note we investigate arithmetic properties of values of the Tschakaloff function $T_{q}(z)=\sum_{\nu=0}^{\infty} q^{-\nu(\nu+1) / 2} z^{\nu},|q|>1$. One of the open problems is proving linear independence of the values of $T_{q}(z)$ with different $q$. The only result obtained in this direction in [1] is very restrictive. We refer an interested reader to the survey [2] for an account of known linear and algebraic independence results for values of Tschakaloff and other $q$-series.

Theorem 1. Let $t_{1}$ and $t_{2}$ be distinct positive integers, and let $q \in \mathbb{Z} \backslash\{0, \pm 1\}$ and $\alpha \in \mathbb{Q} \backslash\{0\}$ be multiplicatively independent ${ }^{1}$. Then the numbers $1, T_{q^{t_{1}}}(\alpha)$, and $T_{q^{t_{2}}}(\alpha)$ are linearly independent over $\mathbb{Q}$.

Note that the function $f_{t}(z)=T_{q^{t}}\left(\alpha z^{t}\right), t \in \mathbb{N}$, satisfies the functional equation $\alpha z^{t} f_{t}(z)=f_{t}(q z)-1$. To prove Theorem 1 we use essentially the method from [3] applied to the functions $g_{i}(z)=f_{t_{i}}\left(z^{m_{i}}\right), i=1,2, m_{i} \in \mathbb{N}$, which satisfy $\alpha_{i} z^{s_{i}} g_{i}(z)=$

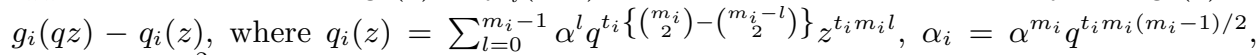
and $s_{i}=t_{i} m_{i}^{2}$. The scaling $z \mapsto z^{m_{i}}$ is a new and crucial ingredient of our construction. There are infinitely many pairs $\left(m_{1}, m_{2}\right) \in \mathbb{N}^{2}$ such that

$$
\left|s_{1}-s_{2}\right|=\left|t_{1} m_{1}^{2}-t_{2} m_{2}^{2}\right| \leqslant c_{0}
$$

with a fixed $c_{0}>0$ (if $\tau=\sqrt{t_{1} / t_{2}}$ is rational then we may even take $c_{0}=0$, while if $\tau$ is irrational then such pairs are obtained, e.g., by using the convergents of the continued fraction expansion of $\tau)$. After the choice of a pair $\left(m_{1}, m_{2}\right)$ satisfying (1) we index our functions $g_{1}(z)$ and $g_{2}(z)$ in such a way that $s_{1} \leqslant s_{2} \leqslant s_{1}+c_{0}$.

Using the Dirichlet box principle we construct Padé-type approximations of the second kind to the functions $g_{1}(z)$ and $g_{2}(z)$.

Lemma 1. Given $\varepsilon, 0<\varepsilon<1 / 2$, and a positive integer $n$, there exist a polynomial $P(z)=\sum_{j=0}^{n} p_{j} z^{j} \in \mathbb{Z}[z]$, not identically zero, and polynomials $Q_{i}(z) \in \mathbb{Q}[z], \operatorname{deg} Q_{i} \leqslant n$, $i=1,2$, such that the following conditions are satisfied:

(i) $\left|p_{j}\right| \leqslant|q|^{\lambda n^{2} / M+O(n)}$, where $\lambda=\lambda(\varepsilon)=(1-2 \varepsilon)(3-2 \varepsilon)^{2} /(8 \varepsilon)$ and $M=2 s_{1}$;

(ii) the coefficients of the polynomials $Q_{i}(z)$ become integers after multiplication by $q^{n^{2} / M+O(n)}$

(iii) if $R_{i}(z)=P(z) g_{i}(z)-Q_{i}(z), i=1,2$, then $\operatorname{ord}_{z=0} R_{i}(z) \geqslant \frac{3}{2} n-\varepsilon n$ and $\left|R_{i}(z)\right| \leqslant$ $|q|^{\lambda n^{2} / M+O(n)}|z|^{\frac{3}{2} n-\varepsilon n}$ whenever $|z| \ll 1$.

Using the functional equation for $g_{i}(z)$ we construct further approximations from Lemma 1. Starting with $R_{i 0}(z)=P_{0}(z) g_{i}(z)-Q_{i 0}(z)=P(z) g_{i}(z)-Q_{i}(z), i=1,2$, we define, for $k=1,2, R_{i k}(z)=\alpha_{i}^{-1} z^{s_{2}-s_{i}} R_{i, k-1}(q z)=P_{k}(z) g_{i}(z)-Q_{i k}(z), i=1,2$. As in [1; Lemma 2] if $s_{1}<s_{2}$, and as in [3; Lemma 3] if $s_{1}=s_{2}$, we now obtain the following non-vanishing lemma.

The work of the second author was supported in part by the INTAS foundation, grant no. 03-51-5070. The grounds of this work were set during the second author's visit in the Department of Mathematical Sciences of the University of Oulu in December 2006. He thanks kindly the staff of the department for its hospitality.

${ }^{1}$ This means that the only integers $i$ and $j$ satisfying $\alpha^{i} q^{j}=1$ are $i=j=0$.

AMS 2000 Mathematics Subject Classification. Primary 11J72; Secondary 11J82.

DOI 10.1070/RM2007v062n01ABEH000000. 
Lemma 2. The determinant

$$
\Delta(z):=\left|\begin{array}{lll}
P_{0}(z) & Q_{10}(z) & Q_{20}(z) \\
P_{1}(z) & Q_{11}(z) & Q_{21}(z) \\
P_{2}(z) & Q_{12}(z) & Q_{22}(z)
\end{array}\right| \neq 0
$$

Comparing the degree and the order at the origin of $\Delta(z)$ we deduce that, for any $\rho \geqslant 0$ there exists an integer $\nu$ such that $\rho n \leqslant \nu \leqslant \rho n+2 \varepsilon n+3 s_{2}$ and $\Delta\left(q^{-\nu}\right) \neq 0$.

Fixing $\varepsilon>0$ in such a way that $\varepsilon<\min \left\{1 / 2,1 /\left(6 c_{0}\right)\right\}$ we choose a pair $\left(m_{1}, m_{2}\right) \in \mathbb{N}^{2}$ satisfying (1) and the inequality $(1+\lambda) / M<\left(1-6 c_{0} \varepsilon\right)(1-2 \varepsilon) /\left(32 c_{0}\right)$, and use the construction above with this pair and with $\rho=\left(1-6 c_{0} \varepsilon\right) /\left(4 c_{0}\right)$. For any $n$ we get linear forms $r_{i k}(\nu)=\alpha_{i}^{\nu} q^{\left(s_{2}-s_{i}\right) \nu(\nu+1) / 2} R_{i k}\left(q^{-\nu}\right)=p_{k}(\nu) g_{i}(1)-q_{i k}(\nu), i=1,2, k=0,1,2$, where the rational coefficients $p_{k}(\nu)$ and $q_{i k}(\nu)$ become integers after multiplication by a positive integer $D_{\nu} \leqslant|q|^{n^{2} / M+n \nu+O(n+\nu)}$. Assuming, contrary to Theorem 1 , that 1 and $g_{i}(1)=f_{t_{i}}(1)=T_{q^{t_{i}}}(\alpha), i=1,2$, are linearly dependent over $\mathbb{Q}$, we have a linear relation $L=h_{0}+h_{1} g_{1}(1)+h_{2} g_{2}(1)=0$ with some integers $h_{0}, h_{1}, h_{2}$ not all zero. Then

$$
0=D_{\nu} p_{k}(\nu) L=D_{\nu}\left(h_{0} p_{k}(\nu)+h_{1} q_{1 k}(\nu)+h_{2} q_{2 k}(\nu)\right)+D_{\nu}\left(h_{1} r_{1 k}(\nu)+h_{2} r_{2 k}(\nu)\right),
$$

and the non-vanishing of the determinant

$$
\left|\begin{array}{lll}
p_{0}(\nu) & q_{10}(\nu) & q_{20}(\nu) \\
p_{1}(\nu) & q_{11}(\nu) & q_{21}(\nu) \\
p_{2}(\nu) & q_{12}(\nu) & q_{22}(\nu)
\end{array}\right|=\left(\alpha_{1} \alpha_{2}\right)^{\nu} q^{\left(2 s_{2}-s_{1}\right) \nu(\nu+1) / 2} \Delta\left(q^{-\nu}\right)
$$

implies the existence of $k \in\{0,1,2\}$ such that the term $D_{\nu}\left(h_{0} p_{k}(\nu)+h_{1} q_{1 k}(\nu)+h_{2} q_{2 k}(\nu)\right)$ in (2) is nonzero. On the other hand, it is an integer, yielding $D_{\nu}\left|h_{1} r_{1 k}(\nu)+h_{2} r_{2 k}(\nu)\right| \geqslant 1$ from (2). This leads to a contradiction since $D_{\nu}\left(h_{1} r_{1 k}(\nu)+h_{2} r_{2 k}(\nu)\right) \rightarrow 0$ as $n \rightarrow \infty$ by Lemma 1 and our choice of the parameters, and Theorem 1 follows.

The result of Theorem 1 remains valid if we replace $\mathbb{Q}$ by an imaginary quadratic field $\mathbb{I}$ and $\mathbb{Z}$ by the ring $\mathbb{Z}_{\mathbb{I}}$ of integers of $\mathbb{I}$. The method in [3] and a slight generalization of the above proof lead to the following general result (cf. [1]).

Theorem 2. Let $q \in \mathbb{Z}_{\mathbb{I}}$ satisfy $|q|>1$. Suppose that $t_{1}, \ldots, t_{l}$ are distinct positive integers, and that a nonzero number $\alpha \in \mathbb{I}$ is multiplicatively independent from $q$. Then the $l+1$ numbers 1 and $T_{q^{t}}(\alpha), k=1, \ldots, l$, are linearly independent over $\mathbb{I}$. Furthermore, if $q$ and $\alpha_{1}, \ldots, \alpha_{l}$ from $\mathbb{I} \backslash\{0\}$ are multiplicatively independent then the numbers 1 and $T_{q^{t} k}\left(\alpha_{k}\right), k=1, \ldots, l$, are linearly independent over $\mathbb{I}$.

To prove this theorem we replace condition (1) by a result obtained from Kronecker's theorem on simultaneous approximations.

We also stress that, due to the quantitative character of the method used in our proofs, it is possible to estimate from below linear forms with integer coefficients involving the numbers in question.

\section{Bibliography}

[1] M. Amou and K. Väänänen, Monatsh. Math. 144:1 (2005), 1-11.

[2] P. Bundschuh, Sūrikaisekikenkyūsho Kōkyūroku 1219 (2001), 110-121. 
[3] K. Väänänen, Math. Ann. 325:1 (2003), 123-136.

\section{K. Väänänen}

Department of Mathematical Sciences,

University of Oulu, Finland

E-mail: kvaanane@sun3.oulu.fi

\section{V. Zudilin (W. Zudilin)}

M. V. Lomonosov Moscow State University;

V. A. Steklov Mathematical Institute,

Russian Academy of Sciences

E-mail: wadim@mi.ras.ru
Presented by A. G. Sergeev

Received 18/JAN/07

Translated by W. ZUDILIN 